\title{
TMT-Based Quantitative Proteomic Analysis Reveals the Crucial Biological Pathways Involved in Self-Incompatibility Responses in Camellia oleifera
}

\author{
Yifan He ${ }^{1,2,3}$, Qianqian Song ${ }^{1,2,3}$, Yuefeng $\mathrm{Wu}^{1,2}$, Shutao $\mathrm{Ye}^{1,2}$, Shipin Chen ${ }^{1,2}$ and \\ Hui Chen 1,2 ,* \\ 1 Forestry College, Fujian Agriculture and Forestry University, Fuzhou 350002, Fujian, China; \\ yifanhe20181@163.com (Y.H.); qianqiansong2018@163.com (Q.S.); yuefengwu01@163.com (Y.W.); \\ yeshutaosir@163.com (S.Y.); Shipin.fjcsp@126.com (S.C.) \\ 2 Forestry College, Oil Tea Research Center of Fujian Province, Fujian Agriculture and Forestry University, \\ Fuzhou 350002, Fujian, China \\ 3 Department of Molecular, Cell \& Developmental Biology, University of California, Los Angeles, CA 90095, \\ USA \\ * Correspondence: huichen@fafu.edu.cn; Tel.: +86-139-5034-3791
}

Received: 4 February 2020; Accepted: 12 March 2020; Published: 14 March 2020

\begin{abstract}
Camellia oleifera is a valuable woody oil plant belonging to the Theaceae, Camellia oil extracted from the seed is an excellent edible oil source. Self-incompatibility (SI) in C. oleifera results in low fruit set, and our knowledge about the mechanism remains limited. In the present study, the Tandem mass tag (TMT) based quantitative proteomics was employed to analyze the dynamic change of proteins response to self- and cross-pollinated in C. oleifera. A total of 6,616 quantified proteins were detected, and differentially abundant proteins (DAPs) analysis identified a large number of proteins. Combined analysis of differentially expressed genes (DEGs) and DAPs of self- and cross-pollinated pistils based on transcriptome and proteome data revealed that several candidate genes or proteins involved in SI of C. oleifera, including polygalacturonase inhibitor, UDP-glycosyltransferase 92A1-like, beta-D-galactosidase, S-adenosylmethionine synthetase, xyloglucan endotransglucosylase/hydrolase, ABC transporter G family member 36-like, and flavonol synthase. Venn diagram analysis identified 11 proteins that may participate in pollen tube growth in C. oleifera. Our data also revealed that the abundance of proteins related to peroxisome was altered in responses to SI in C. oleifera. Moreover, the pathway of lipid metabolism-related, flavonoid biosynthesis and splicesome were reduced in self-pollinated pistils by the Kyoto Encyclopedia of Genes and Genomes (KEGG) pathway analysis. In summary, the results of the present study lay the foundation for learning the regulatory mechanism underlying SI responses as well as provides valuable protein resources for the construction of self-compatibility C. oleifera through genetic engineering in the future.
\end{abstract}

Keywords: Camellia oleifera; proteomic; pathway; self-incompatibility; TMT

\section{Introduction}

Self-incompatibility (SI) exists in most angiosperms as an evolutionary mechanism that stimulates outcrossing and inhibits inbreeding [1]. As a genetically controlled mechanism, SI provides a high level of heterozygosity by overcoming inbreeding depression in flowering plants [2]. Pollen/pollen tube of self-fertilization was recognized and rejected through evolved genetic systems of expressing the same allelic specificity either with style or ovular vicinity and post-fertilization, resulting in seed set inhibited [3]. In addition to the role of SI in intercellular communication research [3,4], it is also an important feature in agronomy [5,6]. SI could occur in all stages from stigma through ovule, therefore it 
is sure that different plant species might evolve different SI systems [7]. The SI mechanism was revealed in limited planta families because of the extensive properties of SI in the flowering plant [8]. According to genetic characteristics, Self-incompatibility models were commonly classified into gametophytic SI (GSI) and sporophytic SI (SSI) [9]. Mainly well-defined SI models were determined on S-locus. There are no less than two genes on the S-loci, one of them is the male determinate factor, and the other one is the female determinate factor [10]. SSI of Brassicaceae is controlled by the stigma receptor kinase $[11,12]$ via interaction with the pollen coat cysteine-rich protein $[13,14]$ and is then phosphorylated intracellularly $[15,16]$. In Rosaceae, Solanaceae, and Plantaginaceae, GSI was accomplished by male determinate factor S-locus F-box protein/S haplotype-specific F-box protein gene (SLF/SFB) and female determinate factor S-RNase [17-19]. Other genes except S-locus were still believed to play important roles in SI [20]. In the Papaveraceae, programmed cell death (PCD) contributed to GSI through phosphorylation, ubiquitination, and calcium-dependent signaling, resulting in the termination of pollen tube growth [21]. Late self-incompatibility (LSI) is defined based on the ovary of which inhibition happened, thus different from the other two SI models [22]. The mechanism of LSI is still unclear although LSI is more widespread in the flowering plant because of its evolution position $[8,9]$. LSI could occur at both the pre- and post-fertilization phases depending on different plant species [23]. Bottleblock of revealing the molecular mechanism exists on these long life span LSI plants because of the difficulty in conducting the molecular genetic test $[8,24]$. Nevertheless, several kinds of research confirmed the genetic basis of LSI is hypothesized to be gametophytic $[23,25,26]$. It has been demonstrated that the LSI mechanism in some plants was polygenic and polymorphic according to crossing experiments $[9,25,27]$. Unfortunately, no LSI determinate genes were found to date which prevents our understanding of LSI.

Camellia oleifera Abel. is a valuable oil tree species native to China, famous for its extensive utilization and long history of cultivation [28]. C. oleifera is grown principally for its seed and is utilized to provide the Camellia oil. The best-treasured product of C. oleifera is Camellia oil, which is known as "eastern olive oil" as a result of its high quality [29]. SI in C. oleifera usually results in a low rate of seed set, which limits the yield. Hence, it is necessary to reveal the molecular mechanism of SI in C. oleifera to improve strategies for breeding to guide the improvement of breeding strategies to enhance the yield in the future. In C. oleifera, recent evidence suggests that self-fertilized pollen can form pollen tubes and growth down through the style, but the self-fertilized pollen fails to complete double fertilization in the ovary [6,30]. YIFAN et al. compared the transcriptome of self- and cross-pollinated pistil and found several candidate genes that may participate in C. oleifera SI [30]. However, knowledge about the molecular mechanism of SI in C. oleifera is still limited to date. In the present study, tandem mass $\operatorname{tag}$ (TMT) based quantitative proteomics was employed to analyze the dynamic change of proteins response to self- and cross-pollination in C. oleifera, which could provide us more information on the protein level about SI response.

\section{Results}

\subsection{Identified Protein Overview}

TMT-based quantitative proteomics was performed to obtain the dynamic change of proteins between self- and cross-pollination pistils in C. oleifera. After quality inspection, obtaining a total of 318,969 (54,545 matched) spectra, of which 33,088 were identified peptides ( 31,071 unique peptides) and 7666 were identified proteins (6616 quantified proteins) (Table 1). The average peptides mass error was less than $10 \mathrm{ppm}$, suggesting MS data has very high mass accuracy (Figure 1a). The majority of the peptides identified were 7 to 21 amino acid residues in length (Figure 1b), which indicated that those samples satisfied the requiring criteria. Supplementary Table S1 showed the identified protein detail information. 
Table 1. Detail information of the identified proteins overview.

\begin{tabular}{cccccc}
\hline $\begin{array}{c}\text { Total } \\
\text { Spectrums }\end{array}$ & $\begin{array}{c}\text { Matched } \\
\text { Spetrums }\end{array}$ & Peptides & $\begin{array}{c}\text { Unique } \\
\text { Peptides }\end{array}$ & $\begin{array}{c}\text { Identified } \\
\text { Proteins }\end{array}$ & $\begin{array}{c}\text { Quantifiable } \\
\text { Proteins }\end{array}$ \\
\hline 318,969 & 54,545 & 33,088 & 31,071 & 7666 & 6616 \\
\hline
\end{tabular}
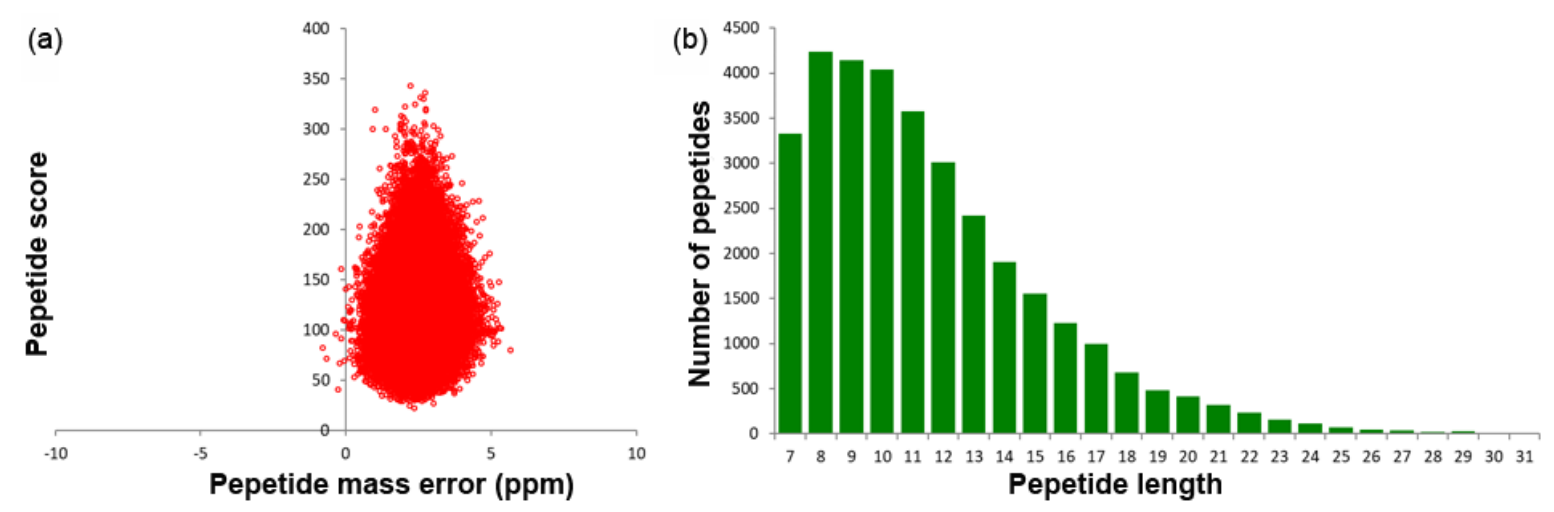

Figure 1. MS identified information based on proteomics analysis: (a) Average peptide mass error; (b) All identified peptides length distribution.

\subsection{Protein Annotation}

Identified protein annotation was performed based on several public databases, containing Gene Ontology (GO) terms, Kyoto Encyclopedia of Genes and Genomes (KEGG) pathways, Protein domain, and Subcellular localization. Detail of information on the identified protein is presented in Supplementary Table S2.

\subsection{Identification and Analysis of Differentially Abundant Proteins}

Differentially abundant proteins (DAPs) were those meeting the criteria (fold change $\geq 1.2$ and $P<0.05)$ under comparison of CP48 vs. CP65, CP48 vs. CP75, SP48 vs. SP65, SP48 vs. CP75, CP48 vs. SP48, CP65 vs. SP65, and CP75 vs. SP75. Within cross-pollination, 212 DAPs were identified in $\mathrm{CP} 48$ vs. CP65, 122 of which were up-regulated and 90 of which were down-regulated; under CP48 vs. CP75, 850 DAPs were identified, 489 of them were up-regulated and 361 of them were down-regulated (Figure 2a). Within self-pollination, 267 DAPs were identified in SP48 vs. SP65, 135 of which were up-regulated and 132 of which were down-regulated; under SP48 vs. SP75, among the 184 DAPs identified, 97 of them were up-regulated and 87 of them were down-regulated (Figure 2a). In total, 227, 260, and 625 DAPs were identified in CP48 vs. SP48, CP65 vs. SP65, and CP75 vs. SP75, respectively. Under CP48 vs. SP48, 227 DAPs were identified, 114 of them were up-regulated and 113 of them were down-regulated. Among the 260 identified DAPs under CP65 vs. SP65, 142 proteins were up-regulated and 118 proteins were down-regulated. Of 625 DAPs identified in SP75 compared with CP75, 366 proteins were up-regulated and 259 proteins were down-regulated (Figure 2b).

Venn diagram analysis was created under CP48 vs. CP65, CP48 vs. CP75, SP48 vs. SP65, and SP48 vs. CP75 (Figure 2c). Eleven proteins were commonly differentially abundant in all these compared groups, which may primarily participate in pollen tube growth in C. oleifera. In addition, to identify the common and specifically differentially abundant proteins participating in the SI mechanism, a Venn diagram was generated within CP48 vs. SP48, CP65 vs. SP65, and CP75 vs. SP75 (Figure 2d). It is shown that 132, 137, and 480 proteins were differentially abundant in CP48 vs. SP48, CP65 vs. SP65, and CP75 vs. SP75, respectively. A total of 21 common differentially abundant proteins were involved in all three compared groups. 
(a)

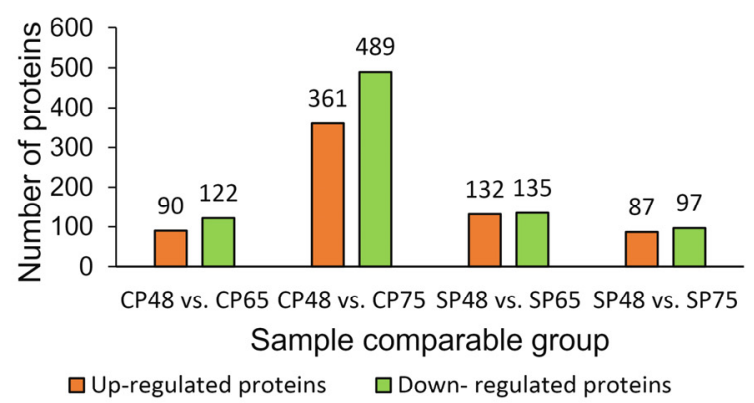

(c)

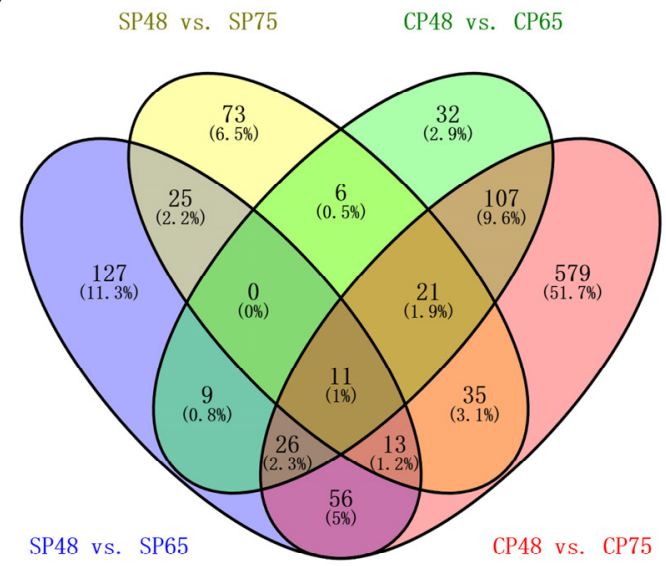

(b)

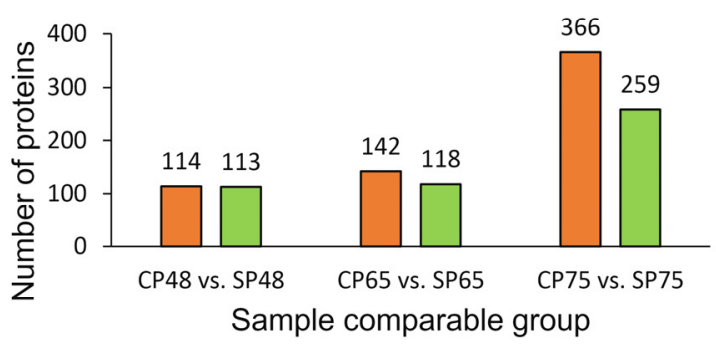

$\square$ Up-regulated proteins $\quad$ DDown-regulated proteins

(d)

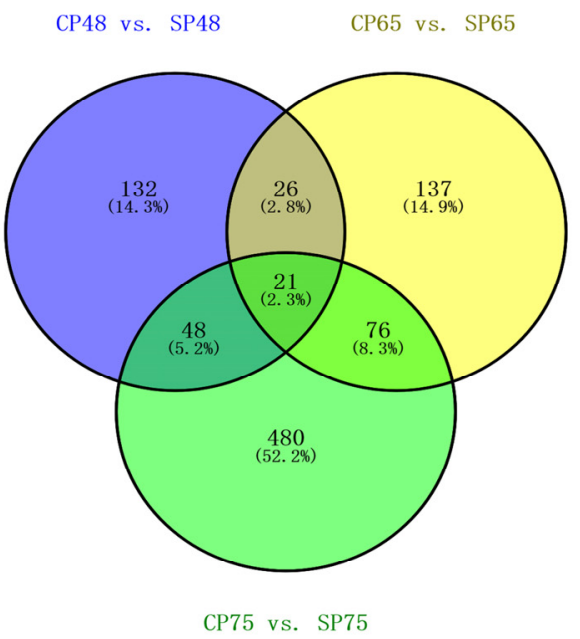

Figure 2. Differentially abundant proteins (DAPs) in all compared groups: (a) The information about DAPs was present in the compared groups of CP48 vs. CP65, CP48 vs. CP75, SP48 vs. SP65, and SP48 vs. CP75. (b) The information about DAPs was present in the compared groups of SP48 vs. CP48, SP65 vs. CP65, and SP75 vs. CP75. (c) DAPs were analyzed by the Venn diagram in the compared groups of CP48 vs. CP65, CP48 vs. CP75, SP48 vs. SP65, and SP48 vs. CP75. (d) DAPs were analyzed by the Venn diagram in the compared groups of SP48 vs. CP48, SP65 vs. CP65, and SP75 vs. CP75. CP48 represents $48 \mathrm{~h}$ after cross-pollination, CP65 represents $65 \mathrm{~h}$ after cross-pollination, CP75 represents $75 \mathrm{~h}$ after cross-pollination, SP48 represents $48 \mathrm{~h}$ after self-pollination, SP65 represents $65 \mathrm{~h}$ after self-pollination, and SP75 represents $75 \mathrm{~h}$ after self-pollination.

\subsection{GO Enrichment Analysis of Pistils between Self- and Cross-Pollinated in C. oleifera}

GO enrichment analysis showed that the DAPs including up-regulated proteins and down-regulated proteins between CP48 and CP65 were classified into 46 functional terms (Supplementary Figure S3); DAPs including up-regulated proteins and down-regulated proteins of CP48 vs. CP75 were classified into 52 GO terms (Supplementary Figure S4), while DAPs including up-regulated proteins and down-regulated proteins of SP48 vs. SP65 were classified into 40 GO terms (Supplementary Figure S5); 33 GO terms were obtained based on the DAPs including up-regulated proteins and down-regulated proteins of SP48 vs. SP75 (Supplementary Figure S6). For cellular components, intracellular non-membrane-bounded organelle, non-membrane-bounded organelle, ribonucleoprotein complex, intracellular ribonucleoprotein complex, and ribosome were commonly found in all four compared groups; for molecular function, structural molecule activity was commonly found in all four compared groups; for biological processes, oxidation-reduction process was commonly found in all four compared groups (Table 2). The results indicated that those common GO terms might be related to the pollen tube growth in the pistil of $C$. oleifera. 
Table 2. Common GO terms under CP48 vs. CP65, CP48 vs. CP75, SP48 vs. SP65, and CP48 vs. CP75.

\begin{tabular}{cc}
\hline Cellular Component & \\
\hline & intracellular non-membrane-bounded organelle \\
\hline non-membrane-bounded organelle \\
\hline ribonucleoprotein complex \\
\hline Molecular Function & intracellular ribonucleoprotein complex \\
\hline Biological Process & ribosome \\
\hline
\end{tabular}

GO enrichment analysis also revealed that the DAPs including up-regulated proteins and down-regulated proteins between CP48 and SP48 were classified into 34 GO terms (Supplementary Figure S7), while DAPs including up-regulated proteins and down-regulated proteins of CP65 vs. SP65 were classified into 39 GO terms (Supplementary Figure S8); 52 GO terms were created based on the DAPs including up-regulated proteins and down-regulated proteins of CP75 vs. SP75 (Supplementary Figure S9). For cellular components, the DAPs were predominantly distributed in the non-membrane-bounded organelle, intracellular organelle, and intracellular ribonucleoprotein complex between CP48 and SP48; the DAPs of CP65 vs. SP65 were principally enriched in the extracellular region; the DAPs were mainly distributed in chromatin, chromosomal part, and intracellular non-membrane-bounded organelle under CP75 vs. SP75. For molecular function, the DAPs primarily found in structural molecule activity, acyl-CoA dehydrogenase activity, and oxidoreductase activity, acting on the $\mathrm{CH}-\mathrm{CH}$ group of donors under $\mathrm{CP} 48$ vs. SP48; the DAPs largely found in methionine adenosyltransferase activity, lyase activity, and Phosphoenolpyruvate carboxykinase (ATP) activity under CP65 vs. SP65; the DAPs of CP75 vs. SP75 essentially found in enzyme inhibitor activity, catalytic activity, and ATP activity. For biological processes, the DAPs of CP48 vs. SP48 generally participated in the oxidation-reduction process, lipid transport, and cell wall macromolecule catabolic process. The DAPs of CP65 vs. SP65 mainly participated in lipid transport and metal ion homeostasis; the DAPs mostly participated in chromatin assembly or disassembly, single-organism metabolic process, and single-organism carbohydrate metabolic process under CP75 vs. SP75.

\subsection{KEGG Pathway Enrichment Analysis of Self- and Cross Pollinated Pistils in C. Oleifera}

KEGG enrichment analysis showed that the DAPs between CP48 and CP65 were largely enriched in the pathway of ribosome (ath03010), biosynthesis of secondary metabolites (ath01110) and peroxisome (ath04146) (Supplementary Figure S10); DAPs between CP48 and CP75 were primarily enriched in the pathway of ribosome (ath03010), spliceosome (ath03040), and phenylalanine metabolism (ath00360) (Supplementary Figure S11); DAPs between SP48 and SP65 were predominantly enriched in the pathway of flavonoid biosynthesis (ath00941), phenylpropanoid biosynthesis (ath00940), and stilbenoid, diarylheptanoid, and gingerol biosynthesis (ath00945) (Supplementary Figure S12); DAPs between SP48 and SP75 were essentially enriched in the pathway of flavonoid biosynthesis (ath00941), phenylpropanoid biosynthesis (ath00940), and biosynthesis of secondary metabolites (ath01110) (Supplementary Figure S13).

KEGG enrichment analysis also showed that the DAPs between CP48 and SP48 were mostly enriched in the pathway of ribosome (ath03010), peroxisome (ath04146), and alpha-linolenic acid metabolism (ath00592) (Figure 3a); DAPs between CP65 and SP65 were largely enriched in the pathway of flavonoid biosynthesis (ath00941), biosynthesis of secondary metabolites (ath01110), and amino sugar and nucleotide sugar metabolism (ath00520) (Figure 3b); DAPs between CP75 and SP75 were primarily 
enriched in the pathway of phenylpropanoid biosynthesis (ath00940), biosynthesis of secondary metabolites (ath01110), and flavonoid biosynthesis (ath00941) (Figure 3c).

(a)

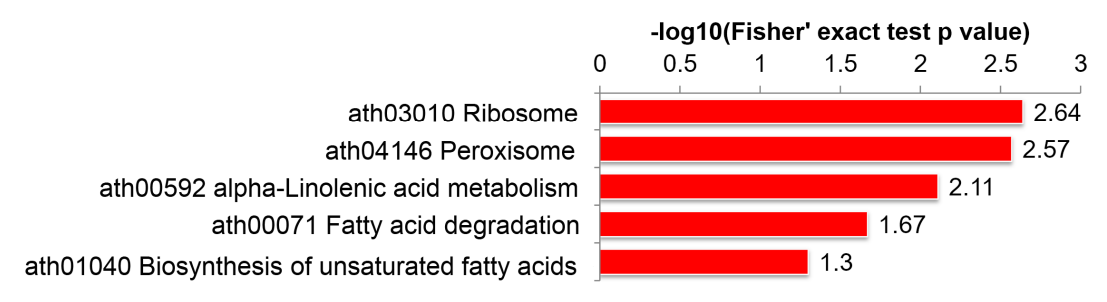

(b)

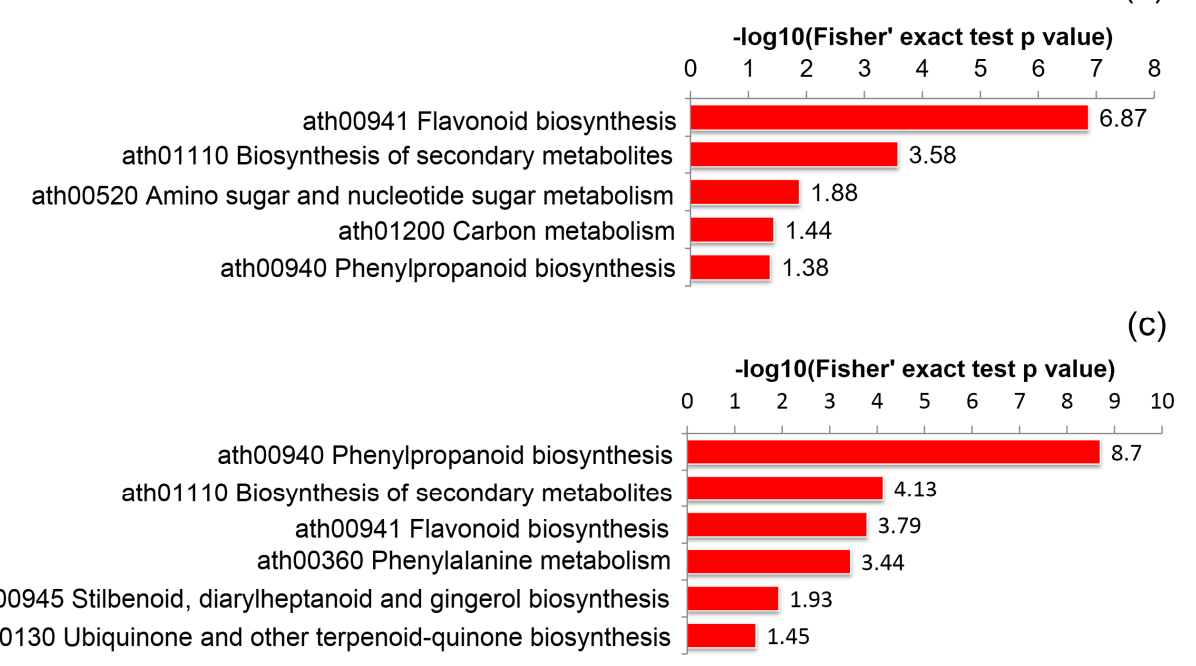

Figure 3. KEGG pathway enrichment analysis of pistils between self- and cross-pollinated in C. oleifera: (a) KEGG pathway enrichment analysis of pistils at $48 \mathrm{~h}$ after self- and cross-pollinated in C. oleifera; (b) KEGG pathway enrichment analysis of pistils at $65 \mathrm{~h}$ after self- and cross-pollinated in C. oleifera; (c) KEGG pathway enrichment analysis of pistils at $75 \mathrm{~h}$ after self- and cross-pollinated in C. oleifera.

\subsection{Protein Domain Enrichment Analysis of Pistils between Self-and Cross-Pollinated in C. oleifera}

Protein domain enrichment analysis showed that the DAPs between CP48 and CP65 were essentially enriched in AMP-dependent synthetase/ligase, Acyl-CoA dehydrogenase/oxidase C-terminal, and AMP-binding enzyme C-terminal domain (Supplementary Figure S14); DAPs between CP48 and CP75 were largely enriched in L-Aspartase-like, RNA helicase, DEAD-box type, Q motif, and Peptidase C1A, papain C-terminal (Supplementary Figure S15); DAPs between SP48 and SP65 were mostly enriched in L-Aspartase-like, Fumarase/histidase, N-terminal, and Alpha crystallin/Hsp20 domain (Supplementary Figure S16); DAPs between SP48 and SP75 were predominantly enriched in SGNH hydrolase-type esterase domain, GDSL lipase/esterase, and Isopenicillin N synthase-like (Supplementary Figure S17).

Protein domain enrichment analysis also showed that the DAPs between CP48 and SP48 were enriched in Bifunctional inhibitor/plant lipid transfer protein/seed storage helical domain, AMP-dependent synthetase/ligase, and Acyl-CoA oxidase/dehydrogenase, central domain (Supplementary Figure S18); DAPs between CP65 and SP65 were enriched in Alpha crystallin/Hsp20 domain, Isopenicillin N synthase-like, and Oxoglutarate/iron-dependent dioxygenase (Supplementary Figure S19); DAPs between CP75 and SP75 were enriched in L-Aspartase-like, Linker histone H1/H5, domain H15, and Pectinesterase inhibitor domain (Supplementary Figure S20). 
2.7. Venn Analysis of DEGs and DAPs of Self- and Cross Pollinated Pistils in C. oleifera Based on Transcriptome and Proteome Data

To better understand the mechanism of SI in C. oleifera, Venn analysis was completed based on the previous transcriptome data [30], which have equivalent comparisons of self- and cross-pollination samples collected at the same time. After $48 \mathrm{~h}$ of self- and cross-pollinated, 18 DEGs or DAPs were found differentially expressed or differentially abundant in pistils (Figure 4a); 30 DEGs or DAPs were found differentially expressed or differentially abundant in pistils after $65 \mathrm{~h}$ of self- and cross-pollinated (Figure 4b); 59 DEGs or DAPs were found differentially expressed or differentially abundant in pistils after $75 \mathrm{~h}$ of self- and cross-pollinated (Figure 4c). These DEGs or DAPs could act important role in the SI of C. oleifera (Supplementary Table S21), including but not limited to polygalacturonase inhibitor (c122207_g1), UDP-glycosyltransferase 92A1-like (c120823_g1), beta-D-galactosidase (c130743_g2), S-adenosylmethionine synthetase (c131722_g2), xyloglucan endotransglucosylase/hydrolase (c130048_g1), ABC transporter G family member 36-like (c133919_g3), and flavonol synthase (c118197_g1). Gene expression is a multistep process, and any step of gene expression may be modulated, from the DNA-RNA transcription step to the post-translational modification of a protein. Our data revealed a low correlation between transcriptome and proteome as well as a much lower dynamic within the proteome compared to the transcriptome. We cannot exclude that this lower dynamic is at least partly due to the different methods used for the respective transcriptomic and proteomic samples. For example, detection efficiencies and sensitivity limits for each method are not necessarily identical. Some differences between the transcriptome and proteome might be due to an extremely low abundance of transcripts or proteins. Moreover, we believe that translation regulation and post-transcriptional regulation likely plays a major role in determining pistil protein accumulation under cross-pollination and self-pollination in C. oleifera. It will be interesting to further explore the regulation of gene expression in self-incompatibility in C. oleifera.

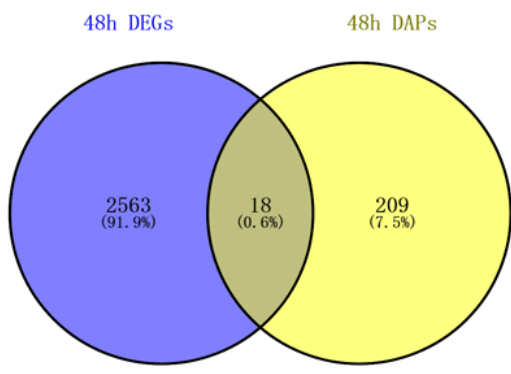

(a)

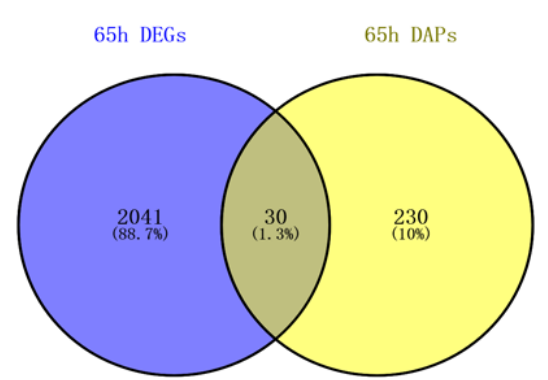

(b)

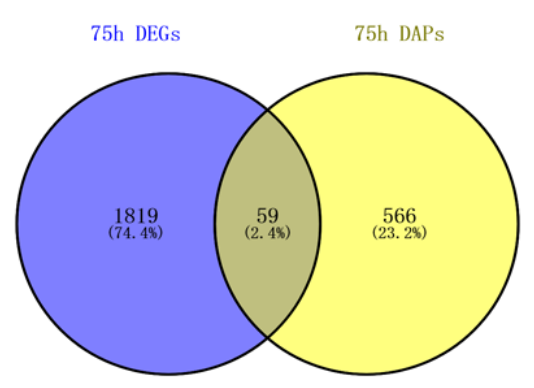

(c)

Figure 4. Venn analysis of differentially expressed genes (DEGs) and DAPs of pistils between self- and cross-pollinated in C. oleifera: (a) Venn analysis of DEGs and DAPs of pistils at $48 \mathrm{~h}$ after self- and cross-pollinated in C. oleifera; (b) Venn analysis of DEGs and DAPs of pistils at $65 \mathrm{~h}$ after self- and cross-pollinated in C. oleifera; (c) Venn analysis of DEGs and DAPs of pistils at $75 \mathrm{~h}$ after self- and cross-pollinated in C. oleifera. 


\section{Discussion}

\subsection{Proteins Participated in Pollen Tube Growth in C. oleifera}

In the present study, eleven proteins were identified and may involve in pollen tube growth in C. oleifera (Table 3). Among them, the protein of c130529_g2 (Ubiquitin-40S ribosomal protein S27a isoform (1) and c82718_g1 (22.7 kDa class IV heat shock protein-like) were down-regulated from $48 \mathrm{~h}$ to $65 \mathrm{~h}$ after pollinated both in self- and cross-pollinated pistils; the other nine proteins were almost up-regulated from $48 \mathrm{~h}$ to $65 \mathrm{~h}$ after pollinated both in self- and cross-pollinated pistils. Sigma factor sigb regulation protein rsbq (c102785_g1) is a hydrolase involved in stress regulation [31]. 9-cis-epoxycarotenoid dioxygenase (NCED) is a vital rate-limiting enzyme in the pathway of abscisic acid (ABA) biosynthetic. Previous studies suggested that ABA biosynthesis could be improved by increased transcript levels of NCED in plants [32,33]. It is interesting to note that the increased protein abundance of c121964_g2 (9-cis-epoxycarotenoid dioxygenase 3 protein family isoform (2) may regulate pollen tube growth through regulating ABA accumulation in C. oleifera pistil. Peroxidase is a widespread enzyme that oxidizes a large number of reducing compounds. Plant class III peroxidases function in the process of oxidation of toxic reductants, salt tolerance, oxygen stress, auxin catabolism, as well as defense responses [34]. Prior studies that have noted the importance of plant class III peroxidases in SI response of Senecio squalidus L.(Asteraceae) [35]. In our work, a class III peroxidase (c124653_g1) was identified and supposed to involve in pollen tube growth in C. oleifera. Phenylpropanoids are identified to act as a critical role in pollen fertility [36]. Phenylalanine ammonia-lyase mediated phenylpropanoid metabolism by catalyzing the first step of the phenylpropanoid skeleton biosynthesis [37]. It is reported that receptor-like kinases (RLKs) mediate pollen tube growth and identification [38]. The current study identified a receptor-like protein kinase HAIKU2 (c135487_g1) which encodes leucine-rich repeat (LRR) kinase, but its function in C. oleifera is unclear. It would be interesting to further explore these identified proteins function in pollen tube growth in C. oleifera.

Table 3. Proteins participated in pollen tube growth in C. oleifera.

\begin{tabular}{ccc}
\hline Protein Accession & Protein Description & Subcellular Localization \\
\hline c102785_g1 & Sigma factor sigb regulation protein rsbq & cytoplasm \\
c121964_g2 & $\begin{array}{c}\text { 9-cis-epoxycarotenoid dioxygenase 3 protein } \\
\text { family isoform 2 }\end{array}$ & mitochondria \\
\hline c124653_g1 & Class III peroxidase & chloroplast \\
\hline c130353_g1 & Alpha-copaene synthase & nucleus \\
\hline c130529_g2 & Ubiquitin-40S ribosomal protein S27a isoform 1 & nucleus \\
\hline c131407_g2 & Phenylalanine ammonia-lyase & chloroplast \\
\hline c132935_g1 & Formamidase-like isoform 1 & nucleus \\
\hline c134412_g1 & Terpene synthase & cytoplasm \\
\hline c134849_g2 & (E)-beta-caryophyllene synthase & cytoskeleton \\
\hline c135487_g1 & Receptor-like protein kinase HAIKU2-like & nucleus \\
\hline c82718_g1 & 22.7 kDa class IV heat shock protein-like & chloroplast \\
\hline
\end{tabular}

\subsection{Peroxisome and Lipid Metabolism Might Act A Key Role in SI Response of C. oleifera}

Peroxisomes are multi-functional organelles critical for plant growth and development [39]. Plant peroxisomes involved in numerous physiological processes, containing phytohormone biosynthesis, lipid catabolism, reactive oxygen species metabolism, and many others [40-42]. It was previously found that Peroxisome morphology9 (APEM9) mediates pollen maturation/germination of which participating in peroxisome biogenesis and function [43]. Interestingly, our data revealed that several genes involved in the pathway of peroxisome, including catalase-3 (c127728_g1), peroxisomal acyl-coenzyme A oxidase 3 (c119854_g1), hypothetical protein (c121573_g1 and c135528_g1), and 
peroxisomal acyl-coenzyme A oxidase 1-like (c119759_g2) were down-regulated $48 \mathrm{~h}$ after self-pollinated compared with cross-pollinated (Supplementary Table S22). Another interesting finding is that a peroxisome biogenesis protein 19-1-like (c114131_g1) was up-regulated $48 \mathrm{~h}$ after self-pollinated compared with cross-pollinated. It was demonstrated that lipids mediate pollen-tube growth by regulating the water flow of pollen [44]. Until recently, there was no genetic evidence to support lipid metabolism is involved in SI response. According to the analysis of the KEGG pathway, the "fatty acid degradation", " $\alpha$-Linolenic acid metabolism", and "biosynthesis of unsaturated fatty acids" were significantly enriched in down-regulated genes $48 \mathrm{~h}$ after self-pollination in C. oleifera (Supplementary Table S22). In particular, genes participated in fatty acid degradation including a peroxisomal acyl-coenzyme A oxidase 3 (c119854_g1), a glyoxysomal fatty acid beta-oxidation multifunctional protein (c121302_g2), and a peroxisomal acyl-coenzyme A oxidase 1-like (c119759_g2) were down-regulated. Energy for pollen germination and pollen tube elongation usually comes from sugar by fatty acid degradation. In Arabidopsis mutants, impairing of fatty acid degradation leads to restriction of pollen tube elongation [45]. It seems that it induces down-regulated fatty acid degradation resulting in energy lacking in SI response in C. oleifera. It must also be mentioned that AMP-dependent CoA ligase (c117849_g1), which is involved in $\alpha$-Linolenic acid metabolism and acyl-coenzyme A thioesterase 8 (c129351_g1), which participated in the unsaturated fatty acids biosynthesis were also down-regulated. As peroxisomes play an essential role in lipid metabolism, combining our research data suggested that these genes involved in peroxisomes and lipid metabolism might cooperate function as candidates for the SI response in C. oleifera.

\subsection{Flavonoids May Negatively Regulate SI Response in C. oleifera}

As a class of secondary metabolites, flavonoids can be divided into flavonols, flavones, proanthocyanidins, anthocyanins, and isoflavones [46-48]. Flavonoids are essential for plant development and stress tolerance [49-51]. The roles of flavonoids in plant reproductive processes were demonstrated [52-59]. Previous studies reported that chalcone synthase-deficient plants present self-incompatibility and male sterility to some extent [60]. As previously described, quercetin and kaempferol produced by flavonoids in tobacco anthers could stimulate pollen tube growth as well as seed development [56]. Previous studies also reported that pollen tubes of flavonol-deficient Petunia show remarkable changes in wall structure resulting in tube disruption [57]. Interestingly, it was found that the abundances of proteins involved in the flavonoid biosynthesis were reduced $75 \mathrm{~h}$ after self-pollinated compared with cross-pollinated (Figure 5). It is important to point out that a total of 8 DAPs involved in flavonoid biosynthesis were decreased $75 \mathrm{~h}$ after pollination, including chalcone synthase (CHS), flavanone 3-hydroxylase (F3H), flavanol synthase (FLS), dihydroflavonol4-reductase (DFR), and anthocyanidin reductase (ANR) (Supplementary Table S23). This decrease in flavonoid enzymes suggests that flavonoids should be necessary for SI in C. oleifera. These results imply that flavonoids may behave as signal molecules negatively regulating SI response in C. oleifera. 


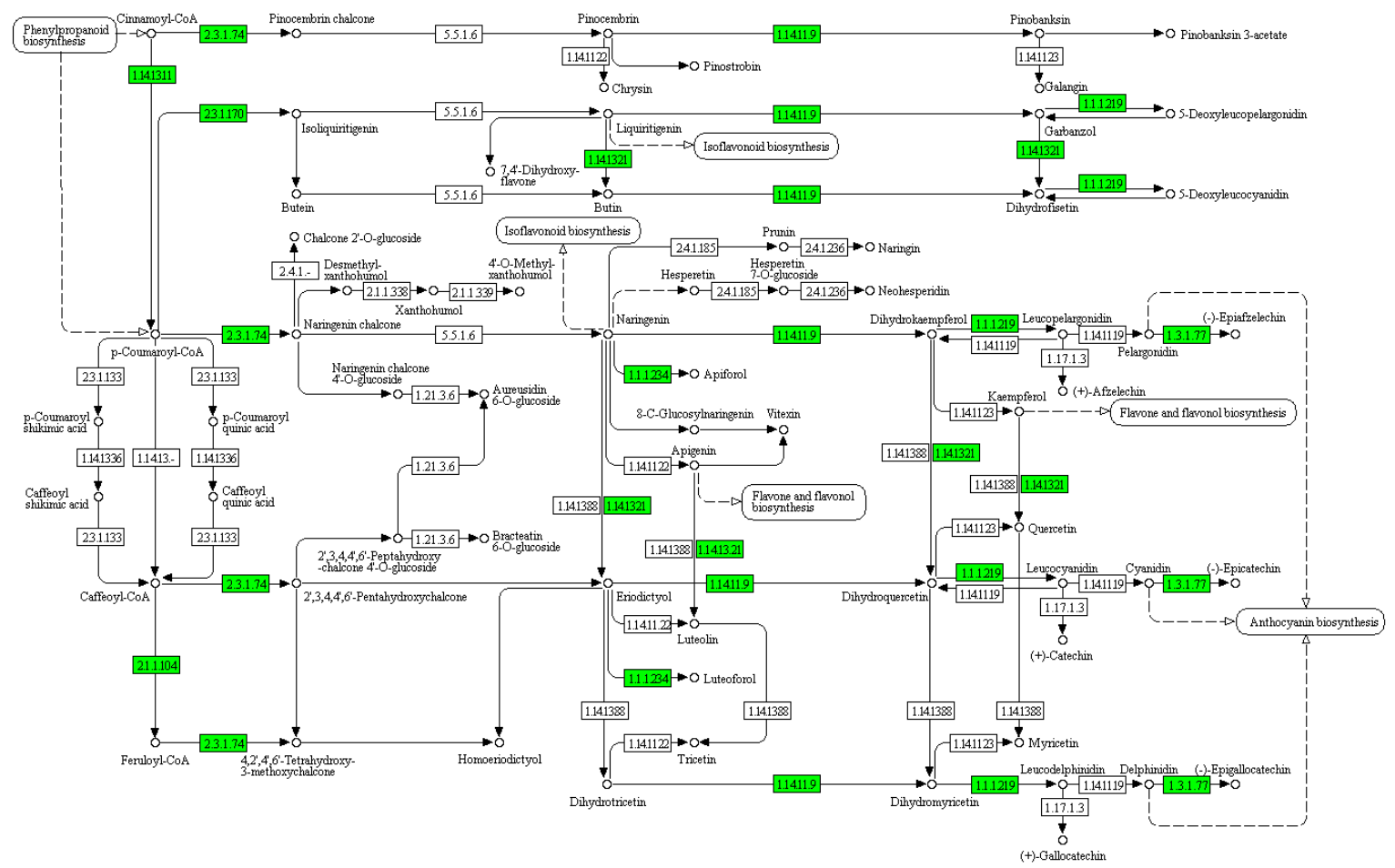

Figure 5. KEGG Pathway analysis of flavonoid biosynthesis $75 \mathrm{~h}$ after self- and cross-pollination in C. oleifera. The green color indicated decreased protein abundances level in self-pollinated pistils compared with cross-pollinated pistils.

\subsection{Splicesome Increased in SI Response in C. oleifera}

RNA processing has been demonstrated to play a crucial role in female gametophyte development [61,62]. Recent evidence suggests that Lachesis (LIS) gene influenced synergid cell fate, which is required for splicing through the U4/U6 spliceosome [63]. In addition, Clotho/Gametophytic factor1 (CLO/GFA1) is an important part of the spliceosome of which are involved in suppressing egg cell fate and gametic cell fate [62]. Additionally, Atropos (ATO) encodes the pre-mRNA splicing factor SF3a60 homolog which is involved in female gametophyte function [62]. Slow walker1 (SWA1) encodes a yeast U3 small nucleolar ribonucleoprotein15 (UTP15) homolog that is essential for pre-RNA processing and the mutant of SWA1 leading to complete female sterility in Arabidopsis [64]. One unanticipated finding was that 25 proteins were identified involved in the pathway of splicesome, and all of them were up-regulated $75 \mathrm{~h}$ after self-pollinated compared with cross-pollinated (Figure 6 and Supplementary Table S24). These data suggested that RNA processing extensively occurred in response to SI in C. oleifera. 


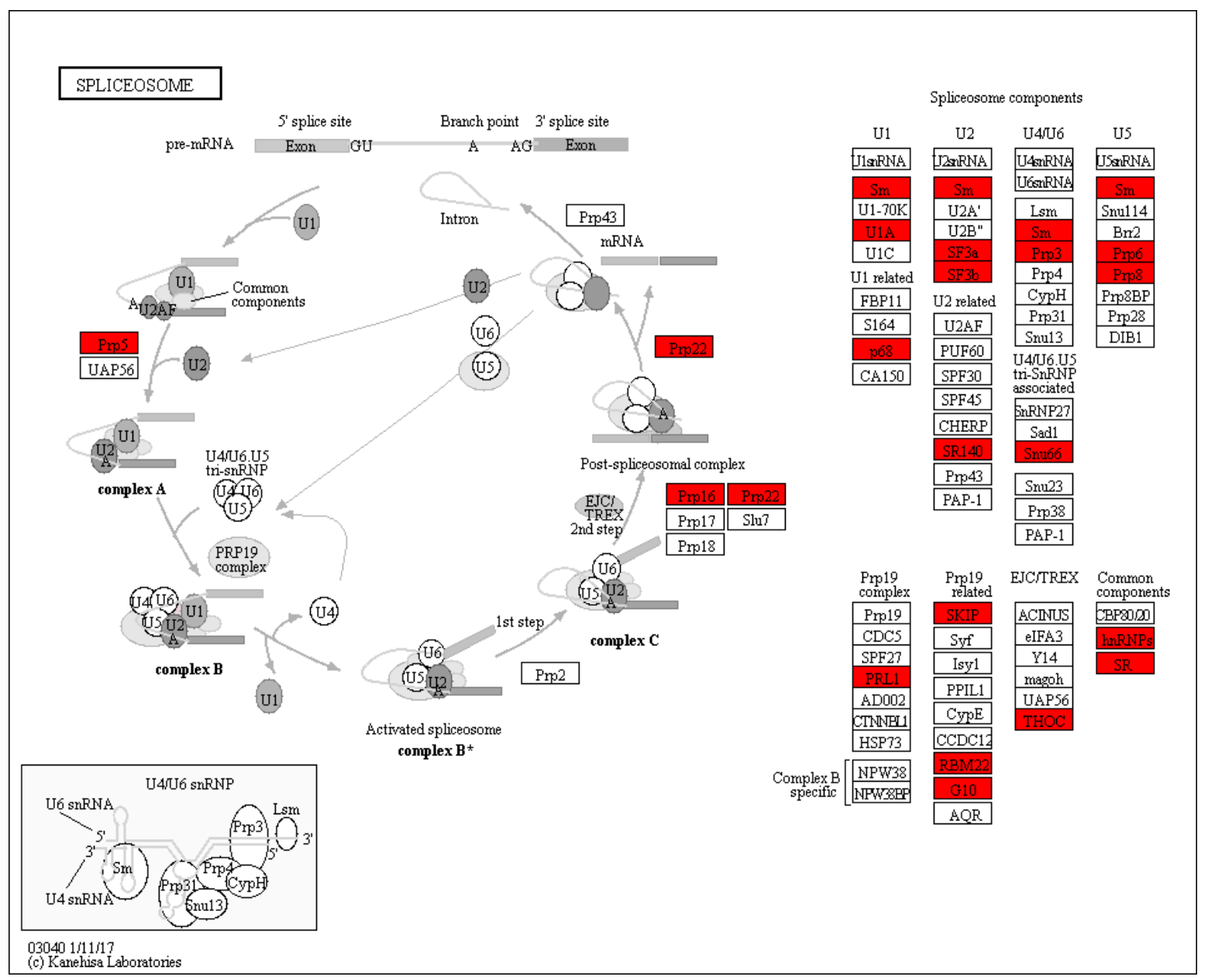

Figure 6. KEGG Pathway analysis of splicesome $75 \mathrm{~h}$ after self- and cross-pollination in C. oleifera. The red color indicated increased protein abundances level in self-pollinated pistils compared with cross-pollinated pistils.

\subsection{Hypothetical Model Occurring in SI Response in C. oleifera}

Contrary to expectation, the female determinate factor S-RNase and the male determinate factor S-locus F-box protein of gametophytic self-incompatibility was not involved in SI response in this study. It seems the SI mechanism of C. oleifera is different from those that have been revealed in the Rosaceae, Solanaceae, and Plantaginaceae $[18,19]$. Based on the results presented in this study, we propose a model for the SI responsive pathways in C. oleifera. According to this model, self-pollination induced alterations in the pathway of peroxisome, and down-regulated lipid metabolism, which resulted in the energy lacking. In the meantime, flavonoids were synthesis decreased, and RNA processing events increased later after self-pollinated. These changes altogether lead to SI in C. oleifera.

\section{Materials and Methods}

\subsection{Plant Materials}

The materials used in the present study include two C. oleifera cultivars of "Min 43" and "Min 48". Then, 25-year-old trees with $2.5 \mathrm{~m}$ height were mixed plant in Minhou Tongkou Forest Farm $\left(26^{\circ} 05^{\prime} \mathrm{N}\right.$, $119^{\circ} 17^{\prime}$ E) where the altitude is $186 \mathrm{~m}$, Fujian Province, China. 


\subsection{Pollination Treatment}

Pollen of the "Min 43" and "Min 48" cultivars was collected from balloon-stage flowers and stored at $25^{\circ} \mathrm{C}$ for eight hours. Self-pollination (SP) of Min $48 \times$ Min 48 and cross-pollination (CP) of Min $48 \times$ Min 43 treatments were performed from 8:00-11:00 am and 1:00-4:00 pm on sunny days in late November. Min 48 was the cultivar donor of pollens and pistils in the self-pollination experiments. In the cross-pollination experiments, Min 43 was the cultivar donor of pollens and Min 48 was the cultivar donor of pistils. Pistils of $48 \mathrm{~h}, 65 \mathrm{~h}$, and $75 \mathrm{~h}$ after SP and CP were harvest and marked as SP48, $\mathrm{SP} 65$, SP75, CP48, CP65, and CP75. Samples were collected in liquid nitrogen and stored at $-80^{\circ} \mathrm{C}$.

\subsection{Protein Extraction}

We used liquid nitrogen to grind samples into powder and then transfer those powders to a $5 \mathrm{~mL}$ centrifuge tube. Next, adding four volumes of lysis buffer ( $8 \mathrm{M}$ urea, $1 \%$ Triton-100, $10 \mathrm{mM}$ dithiothreitol, and 1\% Protease Inhibitor Cocktail, $3 \mu \mathrm{M}$ TSA, $50 \mathrm{mM}$ NAM, and $2 \mathrm{mM}$ EDTA) to the powder, after that sonicated three times on ice by a high-intensity ultrasonic processor (Scientz, Ningbo, Zhejiang, China). The residual debris was discarded through centrifugation at $20,000 \times g$ for $10 \mathrm{~min}$ at $4{ }^{\circ} \mathrm{C}$. Lastly, precipitated the protein in pre-cold $20 \%$ TCA at $4{ }^{\circ} \mathrm{C}$ for $2 \mathrm{~h}$. The supernatant was discarded after being centrifuged at $12,000 \times \mathrm{g}$ for $3 \mathrm{~min}$ at $4{ }^{\circ} \mathrm{C}$. We used cold acetone to wash the residual precipitate three times. Then we redissolved the protein in $8 \mathrm{M}$ urea, and measured the protein concentration by the BCA protein assay kit (Bio-Rad, Hercules, CA, USA).

\subsection{Trypsin Digestion}

We used $5 \mathrm{mM}$ dithiothreitol to reduce the protein solution at $56^{\circ} \mathrm{C}$ for $30 \mathrm{~min}$ and $11 \mathrm{mM}$ iodoacetamide to alkylate at room temperature in darkness for $15 \mathrm{~min}$ to digestion. After that, we added $100 \mathrm{mM}$ TEAB to a urea concentration of below $2 \mathrm{M}$ to dilute the protein sample. Lastly, we added trypsin at a mass ratio of trypsin to the protein of $1: 50$ to perform the first digestion at $37^{\circ} \mathrm{C}$ overnight, and then a second mass of trypsin to protein was 1:100 to perform a second $4 \mathrm{~h}$ to digest.

\subsection{TMT Labeling}

After trypsin digestion, the peptide was desalted using the Strata X C18 SPE column (Phenomenex, Torrance, CA, USA) and vacuum-dried. The peptide was reconstituted in 0.5 M TEAB and treated with TMT reagent (Thermo Fisher Scientific, Carlsbad, CA, USA). Concisely, one unit of TMT reagent was thawed and reconstituted in acetonitrile. The peptide mixtures were then incubated at room temperature for $2 \mathrm{~h}$ and pooled, desalted, and dried by vacuum centrifugation.

\subsection{HPLC Fractionation}

The trypsin-digested peptides were fractionated by high $\mathrm{pH}$ reverse-phase HPLC using Agilent 300Extend C18 column (5 $\mu \mathrm{m}$ particles, $4.6 \mathrm{~mm}$ ID, $250 \mathrm{~mm}$ length, Agilent, Santa Clara, CA, USA). Firstly, peptides were separated into 60 fractions in a gradient of $8 \%$ to $32 \%$ acetonitrile ( $\mathrm{pH} 9.0$ ) over $60 \mathrm{~min}$. After that, the peptides were combined into 18 fractions and dried by vacuum centrifuging.

\subsection{LC-MS/MS Analysis}

The tryptic peptides were dissolved in solvent A ( $0.1 \%$ formic acid in $2 \%$ acetonitrile) and applied directly loaded onto a reversed-phase analytical column with $15 \mathrm{~cm}$ in length and $75 \mu \mathrm{m}$ in diameter (Thermo Fisher Scientific, Carlsbad, CA, USA). The gradient including an increase from $6 \%$ to $24 \%$ solvent B (0.1\% formic acid in $90 \%$ acetonitrile) over $26 \mathrm{~min}, 24 \%$ to $36 \%$ in $8 \mathrm{~min}$, and climbing to $80 \%$ in $3 \mathrm{~min}$ then holding at $80 \%$ for the last $3 \mathrm{~min}$, all performed on an EASY-nLC 1000 UPLC system (Thermo Fisher Scientific, Carlsbad, CA, USA) at a constant flow rate of $350 \mathrm{~nL} / \mathrm{min}$.

The peptides were processed by NSI source and then analyzed by tandem mass spectrometry (MS/MS) in Orbitrap Fusion (Thermo Fisher Scientific, Carlsbad, CA, USA) coupled to UPLC online. 
The applied electrospray voltage was $2.0 \mathrm{kV}$. The $\mathrm{m} / \mathrm{z}$ scan range was 350 to 1550 for a full scan, and intact peptides were detected in the Orbitrap at a resolution of 60,000. Peptides for MS/MS were then selected using the NCE setting as 28 and the fragments were detected in the Orbitrap at a resolution of 15,000 . The data acquisition mode uses a dependent scanning (DDA) program, that is, after the first scan, the first 20 peptides with the highest signal intensity are chosen to enter the peptide precursor and enter the HCD collision cell in turn, using 35\% of the fragmentation energy. Secondary mass spectrometry was performed in sequence. To improve the effective utilization, set the automatic gain control (AGC) to 5E4, the signal threshold to $5000 \mathrm{ions} / \mathrm{s}$, the maximum injection time to $200 \mathrm{~ms}$, and the dynamic exclusion time of the tandem mass spectrometry scan to 30 seconds to prevent the replication of the parent ion scanning.

\subsection{Database Search}

We used the Maxquant search engine (v.1.5.2.8) to process the resulting MS/MS data. Tandem mass spectra were searched against the C. oleifera transcriptomic database (BioProject ID PRJNA507932) concatenated with the reverse decoy database. Trypsin/P was specified as a cleavage enzyme allowing up to 2 missing cleavages. The minimum length of the peptide was set to 7 amino acid residues; the maximum number of peptide modifications was 5 . In the first search, the mass tolerance for precursor ions was set as $20 \mathrm{ppm}$ and $5 \mathrm{ppm}$ in the main search, and the mass tolerance for fragment ions was set as $0.02 \mathrm{Da}$. Carbamidomethyl on Cys was specified as fixed modification and oxidation on Met was specified as variable modifications. The quantitative method was set to TMT-6plex, and the false discovery rate (FDR) for protein identification and peptide-spectrum match (PSM) identification was set to $1 \%$.

\subsection{Data Bioinformatics Analyses}

\subsubsection{GO Annotation}

GO annotation proteome was derived from the UniProt-GOA database. First, the identified protein ID converted to UniProt ID, and then mapped to the GO IDs through the protein ID. If some identified proteins were not annotated through the UniProt-GOA database, the InterProScan soft will be used to annotated the GO function of the proteins based on the protein sequence alignment method. After that, proteins were classified based on three categories: biological process, cellular component, and molecular function through Gene Ontology annotation.

\subsubsection{Domain Annotation}

We annotated the functional description of the identified protein domains using InterProScan (a sequence analysis application) based on the protein sequence alignment method and used the InterPro domain database.

\subsubsection{KEGG Pathway Annotation}

KEGG database was exploited to annotate the protein pathway. First, the KEGG database description of proteins was annotated using the KEGG online service tools KAAS. After that, we used the KEGG online service tools KEGG mapper to map the annotation results to the KEGG pathway database.

\subsubsection{Subcellular Localization}

We employed wolfpsort which is a subcellular localization predication soft to predict subcellular localization. An updated version of PSORT/PSORT II used by Wolfpsort to predict eukaryotic sequences. 


\subsubsection{Enrichment of Gene Ontology Analysis}

Proteins were divided into three categories by GO annotation: biological process, cellular compartment, and molecular function. For each category, a two-tailed fisher's exact test was utilized to test the enrichment of the differentially accumulated protein against all identified proteins. The GO with a corrected $P$-value $<0.05$ is considered significant.

\subsubsection{Enrichment of Pathway Analysis}

KEGG database was exploited to identify enriched pathways through a two-tailed fisher's exact test to test the enrichment of the DAPs against all identified proteins. The pathway with a corrected $P$-value $<0.05$ was considered significant.

\subsubsection{Enrichment of Protein Domain Analysis}

For each category of proteins, the InterPro database was studied and a two-tailed fisher's exact test was utilized to test the enrichment of the DAPs against all identified proteins. Protein domains with a $P$-value $<0.05$ were considered significant.

\section{Conclusions}

In the present study, TMT-based proteomic analysis was conducted to reveal the protein response to self-pollination and cross-pollination. A total of eleven proteins were identified based on Venn analysis of DAPs and may participate in pollen tube growth in C. oleifera. DAPs including polygalacturonase inhibitor, UDP-glycosyltransferase, beta-D-galactosidase, S-adenosylmethionine synthetase, xyloglucan endotransglucosylase/hydrolase, ABC transporter G family member 36-like, and flavonol synthase were remarkably induced responses to SI. Furthermore, KEGG pathway analysis indicated that the pathway of peroxisome, lipid metabolism-related, flavonoids biosynthesis, and splicesome was associated with SI responses in C. oleifera.

Supplementary Materials: Supplementary Materials can be found at http://www.mdpi.com/1422-0067/21/6/1987/ s1. Table S1. The detailed information of identified proteins. Table S2. Protein annotation. Figure S3. GO enrichment analysis of DAPs between CP48 and CP65. Figure S4. GO enrichment analysis of DAPs between CP48 and CP75. Figure S5. GO enrichment analysis of DAPs between SP48 and SP65. Figure S6. GO enrichment analysis of DAPs between SP48 and SP75. Figure S7. GO enrichment analysis of DAPs between CP48 and SP48. Figure S8. GO enrichment analysis of DAPs between CP65 and SP65. Figure S9. GO enrichment analysis of DAPs between CP75 and SP75. Figure S10. KEGG enrichment analysis of DAPs between CP48 and CP65. Figure S11. KEGG enrichment analysis of DAPs between CP48 and CP75. Figure S12. KEGG enrichment analysis of DAPs between SP48 and SP65. Figure S13. KEGG enrichment analysis of DAPs between SP48 and SP75. Figure S14. Protein domain enrichment analysis of DAPs between CP48 and CP65. Figure S15. Protein domain enrichment analysis of DAPs between CP48 and CP75. Figure S16. Protein domain enrichment analysis of DAPs between SP48 and SP65. Figure S17. Protein domain enrichment analysis of DAPs between SP48 and SP75. Figure S18. Protein domain enrichment analysis of DAPs between CP48 and SP48. Figure S19. Protein domain enrichment analysis of DAPs between CP65 and SP65. Figure S20. Protein domain enrichment analysis of DAPs between CP75 and SP75.

Author Contributions: Conceptualization, Y.H. and H.C.; methodology, S.C.; software, Q.S.; validation, Q.S., Y.W. and S.Y.; formal analysis, Y.H.; investigation, Y.W.; resources, H.C.; data curation, Y.H.; writing-original draft preparation, Y.H.; writing—review and editing, Y.H.; visualization, Y.H.; supervision, H.C.; project administration, H.C.; funding acquisition, H.C. All authors have read and agreed to the published version of the manuscript.

Funding: This research was funded by the Special Fund for Science and Technology Innovation of Fujian Agriculture and Forestry University (KFA17274A), and the Special Fund for Science and Technology Innovation of Fujian Agriculture and Forestry University (KFA17049A), the Fujian Seed Industry Innovation and Industrialization Project (KLB17H04A), the Camellia oleifera Germplasm Innovation and Industrialization Project (ZYCX-LY-2017003), and the Sixth Phase of Forestry Seedling Technology Research Project of Fujian Forestry Department (KLB19H02A).

Acknowledgments: We are very thankful to the Minhou Tongkou Forest Farm for supplying research materials. The authors also appreciate the assistance with the experiment provided by Junjie Chen, Yuyin Zheng, Mengmeng Dong, Yinglin Li, Bin Liu, Xiaohui Wu, and Qinmeng Zeng.

Conflicts of Interest: The authors declare no conflict of interest. 


\section{Abbreviations}

\begin{tabular}{|c|c|}
\hline ABA & Abscisic acid \\
\hline AGC & Automatic gain control \\
\hline ANR & Anthocyanidin reductase \\
\hline APEM9 & Peroxisome morphology9 \\
\hline ATO & Atropos \\
\hline ATP & Phosphoenolpyruvate carboxykinase \\
\hline $\mathrm{CHS}$ & Chalcone synthase \\
\hline $\mathrm{CP} 48$ & $48 \mathrm{~h}$ after cross-pollination \\
\hline CP65 & $65 \mathrm{~h}$ after cross-pollination \\
\hline CP75 & $75 \mathrm{~h}$ after cross-pollination \\
\hline DAPs & Differentially abundant proteins \\
\hline DEGs & Differentially expressed genes \\
\hline DFR & Dihydroflavonol4-reductase \\
\hline FDR & false discovery rate \\
\hline FLS & Flavanol synthase \\
\hline GO & Gene Ontology \\
\hline GSI & Gametophytic SI \\
\hline KEGG & Kyoto Encyclopedia of Genes and Genomes \\
\hline LSI & Late self-incompatibility \\
\hline LRR & Leucine-rich repeat \\
\hline NCED & 9-cis-epoxycarotenoid dioxygenase \\
\hline PCD & Programmed cell death \\
\hline PSM & peptide-spectrum match \\
\hline RLKs & Receptor-like kinase \\
\hline SFB & S haplotype-specific F-box protein gene \\
\hline SI & Self-incompatibility \\
\hline SLF & S-locus F-box protein \\
\hline SSI & Sporophytic SI \\
\hline SWA1 & Slow walker1 \\
\hline SP48 & $48 \mathrm{~h}$ after self-pollination \\
\hline SP65 & $65 \mathrm{~h}$ after self-pollination \\
\hline SP75 & $75 \mathrm{~h}$ after self-pollination \\
\hline TMT & Tandem mass tag \\
\hline UTP15 & U3 small nucleolar ribonucleoprotein15 \\
\hline
\end{tabular}

\section{References}

1. Wheeler, M.J.; De Graaf, B.H.J.; Hadjiosif, N.; Perry, R.M.; Poulter, N.S.; Osman, K.; Vatovec, S.; Harper, A.; Franklin, F.C.H.; Franklin-Tong, V.E. Identification of the pollen self-incompatibility determinant in Papaver rhoeas. Nature 2009, 459, 992-995. [CrossRef]

2. De Nettancourt, D. Incompatibility in angiosperms. Sex. Plant Reprod. 1997, 10, 185-199. [CrossRef]

3. Franklin-Tong, V.E.; Ride, J.P.; Franklin, F.C.H. Recombinant stigmatic self-incompatibility (S-) protein elicits a Ca2+ transient in pollen of Papaver rhoeas. Plant J. 1995, 8, 299-307. [CrossRef]

4. Zhou, Q.; Jia, J.; Huang, X.; Yan, X.; Cheng, L.; Chen, S.; Li, X.; Peng, X.; Liu, G. The large-scale investigation of gene expression in Leymus chinensis stigmas provides a valuable resource for understanding the mechanisms of poaceae self-incompatibility. BMC Genom. 2014, 15, 399. [CrossRef] [PubMed]

5. Zhang, S.; Ding, F.; He, X.; Luo, C.; Huang, G.; Hu, Y. Characterization of the 'Xiangshui' lemon transcriptome by de novo assembly to discover genes associated with self-incompatibility. Mol. Genet. Genom. 2014, 290, 365-375. [CrossRef] [PubMed]

6. Liao, T.; Yuan, D.-Y.; Zou, F.; Gao, C.; Yang, Y.; Zhang, L.; Tan, X.-F. Self-Sterility in Camellia oleifera May Be Due to the Prezygotic Late-Acting Self-Incompatibility. PLoS ONE 2014, 9, e99639. [CrossRef]

7. Takayama, S.; Isogai, A. SELF-INCOMPATIBILITY IN PLANTS. Annu. Rev. Plant Boil. 2005, 56, 467-489. [CrossRef] 
8. Gibbs, P.E. Late-acting self-incompatibility-the pariah breeding system in flowering plants. New Phytol. 2014, 203, 717-734. [CrossRef]

9. Allen, A.M.; Hiscock, S.J.; Franklin-Tong, V.E. Evolution and Phylogeny of Self-Incompatibility Systems in Angiosperms. In Self-Incompatibility in Flowering Plants; Springer: Berlin/Heidelberg, Germany, 2008; pp. 73-101.

10. Zhou, Q.; Zheng, Y. Comparative De Novo Transcriptome Analysis of Fertilized Ovules in Xanthoceras sorbifolium Uncovered a Pool of Genes Expressed Specifically or Preferentially in the Selfed Ovule That Are Potentially Involved in Late-Acting Self-Incompatibility. PLoS ONE 2015, 10, e0140507. [CrossRef]

11. Stein, J.C.; Howlett, B.; Boyes, D.C.; Nasrallah, M.E.; Nasrallah, J.B. Molecular cloning of a putative receptor protein kinase gene encoded at the self-incompatibility locus of Brassica oleracea. Proc. Natl. Acad. Sci. USA 1991, 88, 8816-8820. [CrossRef]

12. Watanabe, M.; Suzuki, G.; Takayama, S.; Franklin-Tong, V.E. Milestones Identifying Self-Incompatibility Genes in Brassica Species: From Old Stories to New Findings. In Self-Incompatibility in Flowering Plants; Springer Science and Business Media LLC: Berlin, Germany, 2008; pp. 151-172.

13. Schopfer, C.R. The Male Determinant of Self-Incompatibility in Brassica. Science 1999, 286, 1697-1700. [CrossRef] [PubMed]

14. Takasaki, T.; Hatakeyama, K.; Suzuki, G.; Watanabe, M.; Isogai, A.; Hinata, K. The S receptor kinase determines self-incompatibility in Brassica stigma. Nature 2000, 403, 913-916. [CrossRef] [PubMed]

15. Murase, K.; Shiba, H.; Iwano, M.; Che, F.-S.; Watanabe, M.; Isogai, A.; Takayama, S. A Membrane-Anchored Protein Kinase Involved in Brassica Self-Incompatibility Signaling. Science 2004, 303, 1516-1519. [CrossRef] [PubMed]

16. Kakita, M.; Murase, K.; Iwano, M.; Matsumoto, T.; Watanabe, M.; Shiba, H.; Isogai, A.; Takayama, S. Two Distinct Forms of M-Locus Protein Kinase Localize to the Plasma Membrane and Interact Directly with S-Locus Receptor Kinase to Transduce Self-Incompatibility Signaling in Brassica rapa. Plant Cell 2007, 19, 3961-3973. [CrossRef]

17. Murfett, J.; Atherton, T.L.; Mou, B.; Gassert, C.S.; McClure, B. S-RNase expressed in transgenic Nicotiana causes S-allele-specific pollen rejection. Nature 1994, 367, 563-566. [CrossRef]

18. Sijacic, P.; Wang, X.; Skirpan, A.L.; Wang, Y.; Dowd, P.E.; McCubbin, A.G.; Huang, S.; Kao, T.-H. Identification of the pollen determinant of S-RNase-mediated self-incompatibility. Nature 2004, 429, 302-305. [CrossRef]

19. McClure, B. New views of S-RNase-based self-incompatibility. Curr. Opin. Plant Boil. 2006, 9, 639-646. [CrossRef]

20. Goldraij, A.; Kondo, K.; Lee, C.B.; Hancock, C.N.; Sivaguru, M.; Vazquez-Santana, S.; Kim, S.; Phillips, T.E.; Cruz-Garcia, F.; McClure, B. Compartmentalization of S-RNase and HT-B degradation in self-incompatible Nicotiana. Nature 2006, 439, 805-810. [CrossRef]

21. Thomas, S.; Franklin-Tong, V.E. Self-incompatibility triggers programmed cell death in Papaver pollen. Nature 2004, 429, 305-309. [CrossRef]

22. Ladoux, T.; Friar, E.A. Late-Acting Self-Incompatibility inIpomopsis tenuifolia(Gray) V. Grant (Polemoniaceae). Int. J. Plant Sci. 2006, 167, 463-471. [CrossRef]

23. Cope, F.W. The mechanism of pollen incompatibility in Theobroma cacao L. Heredity 1962, 17, $157-182$. [CrossRef]

24. Seavey, S.R.; Bawa, K. Late-acting self-incompatibility in angiosperms. Bot. Rev. 1986, 52, 195-219. [CrossRef]

25. Lipow, S.R.; Wyatt, R. Single gene control of postzygotic self-incompatibility in poke milkweed, Asclepias exaltata L. Genetics 2000, 154, 893-907. [PubMed]

26. Sage, T.L.; Bertin, R.I.; Williams, E.G. Ovarian and Other Late-Acting Self-Incompatibility Systems; Springer Science and Business Media LLC: Berlin, Germany, 1994; Volume 2, pp. 116-140.

27. Sage, T.; Williams, E. Structure, ultrastructure, and histochemistry of the pollen tube pathway in the milkweed Asclepias exaltata L. Sex. Plant Reprod. 1995, 8, 257-265. [CrossRef]

28. Yu, Y.; Ren, S.; Tan, K. Study on climatic regionalization and layer and belt distribution of oiltea camellia quality in China. J. Natl. Res. 1999, 14, 123-127.

29. Lee, C.-P.; Yen, G.-C. Antioxidant Activity and Bioactive Compounds of Tea Seed (Camellia oleiferaAbel.) Oil. J. Agric. Food Chem. 2006, 54, 779-784. [CrossRef] 
30. He, Y.; Song, Q.; Chen, S.; Wu, Y.; Zheng, G.; Feng, J.; Yang, Z.; Lin, W.; Li, Y.; Chen, H. Transcriptome analysis of self- and cross-pollinated pistils revealing candidate unigenes of self-incompatibility in Camellia oleifera. J. Hortic. Sci. Biotechnol. 2019, 95, 19-31. [CrossRef]

31. Shahali, Y.; Sutra, J.-P.; Fasoli, E.; D’Amato, A.; Righetti, P.G.; Futamura, N.; Boschetti, E.; Sénéchal, H.; Poncet, P. Allergomic study of cypress pollen via combinatorial peptide ligand libraries. J. Proteom. 2012, 77, 101-110. [CrossRef]

32. Qin, X.; Zeevaart, J.A.D. Overexpression of a 9-cis-Epoxycarotenoid Dioxygenase Gene in Nicotiana plumbaginifolia Increases Abscisic Acid and Phaseic Acid Levels and Enhances Drought Tolerance1. Plant Physiol. 2002, 128, 544-551. [CrossRef]

33. Martínez-Andújar, C.; Ordiz, M.I.; Huang, Z.; Nonogaki, M.; Beachy, R.N.; Nonogaki, H. Induction of 9-cis-epoxycarotenoid dioxygenase in Arabidopsis thaliana seeds enhances seed dormancy. Proc. Natl. Acad. Sci. USA 2011, 108, 17225-17229. [CrossRef]

34. Yoshida, K.; Kaothien-Nakayama, P.; Matsui, T.; Kawaoka, A.; Shinmyo, A. Molecular biology and application of plant peroxidase genes. Appl. Microbiol. Biotechnol. 2003, 60, 665-670. [CrossRef] [PubMed]

35. McInnis, S.M.; Costa, L.M.; Gutierrez-Marcos, J.F.; Henderson, C.A.; Hiscock, S.J. Isolation and characterization of a polymorphic stigma-specific class III peroxidase gene from Senecio squalidus L. (Asteraceae). Plant Mol. Boil. 2005, 57, 659-677. [CrossRef] [PubMed]

36. Taylor, L.P.; Hepler, P.K. POLLEN GERMINATION AND TUBE GROWTH. Annu. Rev. Plant Boil. 1997, 48, 461-491. [CrossRef] [PubMed]

37. Jones, D. Phenylalanine ammonia-lyase: Regulation of its induction, and its role in plant development. Phytochemistry 1984, 23, 1349-1359. [CrossRef]

38. Chai, S.; Ge, F.; Li, S.; Zhang, Y. The journey to glory: Receptor-like kinases in pollen tube growth. Sci. Bull. 2016, 61, 827-831. [CrossRef]

39. Pan, R.; Liu, J.; Hu, J. Peroxisomes in plant reproduction and seed-related development. J. Integr. Plant Boil. 2019, 61, 784-802. [CrossRef]

40. Kaur, N.; Reumann, S.; Hu, J. Peroxisome Biogenesis and Function. Arab. Book 2009, 7, e0123. [CrossRef]

41. Kao, Y.-T.; Gonzalez, K.; Bartel, B. Peroxisome Function, Biogenesis, and Dynamics in Plants. Plant Physiol. 2017, 176, 162-177. [CrossRef]

42. Pan, R.; Satkovich, J.; Chen, C.; Hu, J. The E3 ubiquitin ligase SP1-like 1 plays a positive role in peroxisome biogenesis in Arabidopsis. Plant J. 2018, 94, 836-846. [CrossRef]

43. Li, X.; Li, H.-J.; Yuan, L.; Liu, M.; Shi, D.-Q.; Liu, J.; Yang, W.-C. Arabidopsis DAYU/ABERRANT PEROXISOME MORPHOLOGY9 Is a Key Regulator of Peroxisome Biogenesis and Plays Critical Roles during Pollen Maturation and Germination in Planta. Plant Cell 2014, 26, 619-635. [CrossRef]

44. Wolters-Arts, M.; Lush, W.M.; Mariani, C. Lipids are required for directional pollen-tube growth. Nature 1998, 392, 818-821. [CrossRef] [PubMed]

45. Footitt, S.; Dietrich, D.; Fait, A.; Fernie, A.R.; Holdsworth, M.J.; Baker, A.; Theodoulou, F. The COMATOSE ATP-Binding Cassette Transporter Is Required for Full Fertility in Arabidopsis1. Plant Physiol. 2007, 144, 1467-1480. [CrossRef] [PubMed]

46. Grotewold, E. THE GENETICS AND BIOCHEMISTRY OF FLORAL PIGMENTS. Annu. Rev. Plant Boil. 2006, 57, 761-780. [CrossRef] [PubMed]

47. Mierziak, J.; Kostyn, K.; Kulma, A. Flavonoids as Important Molecules of Plant Interactions with the Environment. Molecules 2014, 19, 16240-16265. [CrossRef]

48. Saito, K.; Yonekura-Sakakibara, K.; Nakabayashi, R.; Higashi, Y.; Yamazaki, M.; Tohge, T.; Fernie, A.R. The flavonoid biosynthetic pathway in Arabidopsis: Structural and genetic diversity. Plant Physiol. Biochem. 2013, 72, 21-34. [CrossRef]

49. Martinez, V.; Mestre, T.C.; Rubio, F.; Girones-Vilaplana, A.; Moreno, D.A.; Mittler, R.; Rivero, R.M. Accumulation of Flavonols over Hydroxycinnamic Acids Favors Oxidative Damage Protection under Abiotic Stress. Front. Plant Sci. 2016, 7, 838. [CrossRef]

50. Schenke, D.; Utami, H.P.; Zhou, Z.; Gallegos, M.-T.; Cai, D. Suppression of UV-B stress induced flavonoids by biotic stress: Is there reciprocal crosstalk? Plant Physiol. Biochem. 2019, 134, 53-63. [CrossRef]

51. Winkel, B.S.J. Biosynthesis of flavonoids and effects of stress. Curr. Opin. Plant Boil. 2002, 5, $218-223$. [CrossRef] 
52. Van Der Meer, I.M.; Stam, M.E.; Van Tunen, A.J.; Mol, J.N.; Stuitje, A.R. Antisense inhibition of flavonoid biosynthesis in petunia anthers results in male sterility. Plant Cell 1992, 4, 253-262.

53. Mo, Y.; Nagel, C.; Taylor, L.P. Biochemical complementation of chalcone synthase mutants defines a role for flavonols in functional pollen. Proc. Natl. Acad. Sci. USA 1992, 89, 7213-7217. [CrossRef]

54. Ylstra, B.; Busscher, J.; Franken, J.; Hollman, P.C.; Mol, J.N.; Van Tunen, A.J. Flavonols and fertilization in Petunia hybrida: Localization and mode of action during pollen tube growth. Plant J. 1994, 6, 201-212. [CrossRef]

55. Vogt, T.; Pollak, P.; Tarlyn, N.; Taylor, L.P. Pollination- or Wound-Induced Kaempferol Accumulation in Petunia Stigmas Enhances Seed Production. Plant Cell 1994, 6, 11-23. [CrossRef] [PubMed]

56. Xu, P.; Vogt, T.; Taylor, L.P. Uptake and metabolism of flavonols during in-vitro germination of Petunia hybrida (L.) pollen. Planta 1997, 202, 257-265. [CrossRef]

57. Derksen, J.; Knuiman, B.; Ylstra, B.; Van Tunen, A.J. Pollen tubes of flavonol-deficient Petunia show striking alterations in wall structure leading to tube disruption. Planta 1999, 207, 575-581. [CrossRef]

58. Miller, K.D.; Guyon, V.; Evans, J.N.S.; Shuttleworth, W.A.; Taylor, L.P. Purification, Cloning, and Heterologous Expression of a Catalytically Efficient Flavonol 3-O-Galactosyltransferase Expressed in the Male Gametophyte ofPetunia hybrida. J. Boil. Chem. 1999, 274, 34011-34019. [CrossRef]

59. Guyon, V.N. Isolation and Characterization of cDNAs Expressed in the Early Stages of Flavonol-Induced Pollen Germination in Petunia. Plant Physiol. 2000, 123, 699-710. [CrossRef]

60. Taylor, L.P.; Jorgensen, R. Conditional Male Fertility in Chalcone Synthase-Deficient Petunia. J. Hered. 1992, 83, 11-17. [CrossRef]

61. Liu, Y.; Yan, Z.; Chen, N.; Di, X.; Huang, J.; Guo, G. Development and function of central cell in angiosperm female gametophyte. Genesis 2010, 48, 466-478. [CrossRef]

62. Moll, C.; Von Lyncker, L.; Zimmermann, S.; Kägi, C.; Baumann, N.; Twell, D.; Grossniklaus, U.; Groß-Hardt, R. CLO/GFA1andATOare novel regulators of gametic cell fate in plants. Plant J. 2008, 56, 913-921. [CrossRef]

63. Gross-Hardt, R.; Kägi, C.; Baumann, N.; Moore, J.M.; Baskar, R.; Gagliano, W.B.; Jürgens, G.; Grossniklaus, U.; Groß-Hardt, R. LACHESIS restricts gametic cell fate in the female gametophyte of Arabidopsis. PLoS Boil. 2007, 5, e47. [CrossRef]

64. Ishida, T.; Maekawa, S.; Yanagisawa, S. The Pre-rRNA Processing Complex in Arabidopsis Includes Two WD40-Domain-Containing Proteins Encoded by Glucose-Inducible Genes and Plant-Specific Proteins. Mol. Plant 2016, 9, 312-315. [CrossRef] [PubMed] 